\title{
2 On growth measurements of abdominal aortic aneurysms using maximally
}

3

4

5

17 Address for Correspondence:

18

19

26 sbaek@egr.msu.edu USA

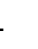

15

6

8

S. Baek, Ph.D.

2457 Engineering Building

Michigan State University

East Lansing, MI 48824

$\mathrm{T}:+1-517-432-3161$

25 F: +1-517-353-1750 \section{inscribed spheres}

${ }^{1}$ Department of Mechanical Engineering, Michigan State University, East Lansing, Michigan 48824, USA

${ }^{2}$ Department of Statistics and Probability, Michigan State University, East Lansing, Michigan 48824,

${ }^{3}$ Department of Electrical and Computer Engineering, Michigan State University, East Lansing, Michigan 48824, USA

${ }^{4}$ Department of Radiology, Seoul National University Hospital, 101 Daehangno, Jongno-gu Seoul, Korea

Department of Mechanical Engineering

27

(Words: 5343) 


\section{Abstract}

29 The maximum diameter, total volume of the abdominal aorta, and its growth rate are usually regarded as

30 key factors for making a decision on the therapeutic operation time for an abdominal aortic aneurysm

31 (AAA) patient. There is, however, a debate on what is the best standard method to measure the diameter.

32 Currently, two dominant methods for measuring the maximum diameter are used. One is measured on the

33 planes perpendicular to the aneurism's central line (orthogonal diameter) and the other one is measured

34 on the axial planes (axial diameter). In this paper, another method called 'inscribed-spherical diameter' is

35 proposed to measure the diameter. The main idea is to find the diameter of the largest sphere that fits

36 within the aorta. An algorithm is employed to establish a centerline for the AAA geometries obtained

37 from a set of longitudinal scans obtained from South Korea. This centerline, besides being the base of the

38 inscribed spherical method, is used for the determination of orthogonal and axial diameter. The growth

39 rate parameters are calculated in different diameters and the total volume and the correlations between

40 them are studied. Furthermore, an exponential growth pattern is sought for the maximum diameters over

41 time to examine a nonlinear growth pattern of AAA expansion both globally and locally. The results

42 present the similarities and discrepancies of these three methods. We report the shortcomings and the

43 advantages of each method and its performance in the quantification of expansion rates. While the

44 orthogonal diameter measurement has an ability of capturing a realistic diameter, it fluctuated. On the

45 other hand, the inscribed sphere diameter method tends to underestimate the diameter measurement but

46 the growth rate can be bounded in a narrow region for aiding prediction capability. Moreover, expansion

47 rate parameters derived from this measurement exhibit good correlation with each other and with growth

48 rate of volume.

49 In conclusion, although the orthogonal method remains the main method of measuring the diameter of an

50 abdominal aorta, employing the idea of maximally inscribed spheres provides both a tool for generation

51 of the centerline, and an additional parameter for quantification of aneurysmal growth rates. 
Keywords: exponential growth, orthogonal diameter, Korean patients

\section{Introduction}

An abdominal aortic aneurysm (AAA) is the localized enlargement of the abdominal aorta that affects a large part of the elderly population; and the more it dilates, the more it will become prone to rupture that is associated with a high mortality rate. Current treatments involve surgical, and either open or endovascular repair. Unfortunately, the risk of these approaches is also high. Therefore, there is an imperative need to decide whether or not an AAA patient needs a medical intervention. Nonetheless, there is no solid argument regarding the appropriate time for an AAA patient to undergo surgery [1-3]. In clinical practice, aneurysms with diameters larger than 50 or $55 \mathrm{~mm}$ are considered for surgical intervention [4-10]. There are, however, uncertainties about the methods of measurement of the diameter [11-14], quantification of dynamic factors $[2,15,16]$, and even sufficiency of the diameter as a predictor for AAA size evolution [1,3,15-19]. These uncertainties have led others to suggest other parameters: the AAA volume, blood pressure, age, sex, and calcium level as predictors for the time of surgery for AAA patients $[1-3,20-22]$.

Previous studies have utilized various ways of measuring the maximum diameter of an AAA and its growth rate. Although investigators have suggested different methods, most of them involve finding the maximum diameter either on an axial plane (“axial diameter") or on a plane orthogonal to aorta's centerline ("orthogonal diameter") $[12,13,16,21,23,24]$. Major concerns associated with measuring the diameter are, however, the accuracy of the estimation and reproducibility of the method [13]. Abada et al. [11] recommended using the maximum anterior posterior or maximum transverse diameter on axial slices. Dugas et al. [13] studied differences of the axial and orthogonal diameter measurements and suggested that the axial diameter measurements overestimate the diameter and that the orthogonal diameter method is more reproducible. Kontopodis et al. [12] illustrated that the median of the differences between the two methods are not high but there are cases where the wide range of differences in measurement possibly affect therapeutic decisions. Those studies proposed that the orthogonal diameter can better represent the 
AAA size than the axial diameters do, while finding that the perpendicular plane to vessel centerline can result in measurement uncertainty. Volume has been introduced as an alternative factor to assess aneurysm development in an AAA patient $[2,12,15,16,18,25,26]$. Raghavan et al. [26] have reported that AAA volume and rupture risk are correlated more strongly than diameter and rupture risk. In their study, to calculate the total volume of an AAA, the aneurysm is axially sliced and the cross sectional area of each axial slice is multiplied by the vertical distance between the centroids of two consecutive slices. Kleinstreuer and Li [2] proposed a severity parameter that integrates alterations of different biomechanical factors (such as maximum diameter and expansion rate) over time by a single value. Recently, Martufi et al. [16] suggested that monitoring only the maximum diameter for surveillance programs may wrongly reflect the expansion related to wall weakening. In their paper, centerline based tools have been introduced to compare the diameter growth might greatly enhance the efficiency of AAA surveillance programs. Additionally, growth of AAAs is measured by means of an exponential growth model. However, computing growth is somewhat of a subjective issue among researchers.

There are simple ways, of course, to define growth rates in different AAA size measurements (cf. [15] and [16]). One approach is quantifying the growth rate by calculating the change in the diameter divided by the time interval between two consecutive images in a linear fashion. Nonetheless, there are multiple practical issues associated with this method such as an inaccuracy due to a relatively small change in diameter over time, the nonlinear nature of the expansion [27], and so forth. Several studies have utilized an exponential growth function for predicting AAA expansion over time [15,16,21,28-30]. Although Martufi's study [16] suggested that an exponential growth parameter can capture AAA's growth, more studies need to be conducted to increase our understanding of an AAA growth pattern; hence, one of the objectives of this study is to examine whether the exponential growth pattern is reasonable so that it can provide a prediction capability for AAA clinical management. 
103 The present study also introduces a new method for the AAA's diameter measurement. This method

104 involves finding the diameters of maximally-inscribed spheres within the geometry, and consequently

105 constructing the centerline using the series spheres' centers. This idea has been widely used for different

106 purposes among researchers [31-33]. We suggest that the proposed definition of the diameter carries

107 useful information related to the size of the AAA, which can be used along with other methods to find the

108 correlations among different geometric parameters and their growth rates. Additionally, this method

109 possibly assists in prediction of the future progression of the disease.

110 To this end, an efficient computational algorithm is developed to compute the inscribed-spherical

111 diameter, and the advantages of using this method are presented. Besides, an exponential growth pattern

112 for maximum diameter is evaluated in the patient group.

\section{2. Methods}

\section{Exponential growth rate}

116 An exponential growth model is a widely accepted growth rate for many biological and physical

117 occurrences. The growth model is proposed based on a nonlinear growth rate $g$ introduced in [16].

$g=(\operatorname{Exp}(12 r)-1) \times 100[\% /$ year $]$

118 where the variable $r$ is measured using a logarithmic growth rate.

$r=\frac{1}{t} \operatorname{Ln}\left(\frac{X^{\text {follow-up }}}{X^{\text {baseline }}}\right)$

119 The quantity $X$ is measured and $t$ is the time interval between two consecutive images in months. To

120 calculate the logarithmic growth rate, the two quantities, $X^{\text {follow-up }}$ and $X^{\text {baseline }}$, are at the same 121 position on the normalized centerline. 
122 Equation (1) combined with equation (2) can be rewritten for the maximum diameter $D$ as below

$$
D^{\text {follow-up }}=D^{\text {baseline }}\left(1+\frac{g}{100}\right)^{\frac{t}{12}}
$$

123 It is equivalent to the following form:

$D^{\text {follow-up }}=D^{\text {baseline }} e^{k t}$

124 where

$k=\ln \left(1+\frac{g}{100}\right) / 12$.

125 Using these equations, an exponential function can provide a curve representing the evolution of the

126 maximal diameter versus time for all the patients. Since the first scan time on monitoring the disease

127 progression of the patients is not the same, each set of data is unfixed with respect to time, without

128 changing the time intervals between two successive images. However, the values measured for diameters

129 are maintained the same. An initial curve is then chosen and the patient's data are moved to match the

130 exponential curve using the least square method. Then another curve is fitted and the process continues

131 iteratively until a certain minimum amount of error is reached. This general exponential curve can be

132 employed to achieve a better understanding of an AAA size and a more accurate prediction of the

133 evolution of the disease.

\section{CT scan data}

135 This study was subject to Internal Review Board approvals at Michigan State University and Seoul 136 National University Hospital.

137 A total of 59 computed tomography (CT) scan data for 14 AAA patients were obtained from Seoul 138 National University Hospital, South Korea. Patients were scanned repeatedly between 3 and 56 months, 
139 and the median was 8 months for all of the follow-up periods. The scans were performed using a $100 \mathrm{KV}$,

$14088 \mathrm{mAs}$ Somatom Sensation $16 \mathrm{CT}$ scanner (Siemens Healthcare, Erlangen, Germany). The slice

141 thickness is $1 \mathrm{~mm}$ and 2D pixel size is $0.641 \mathrm{~mm}$. Further information about the patients is presented in

142 Table 1.

143

144

145

146

147

Table 1. Patients' demographics for AAAs. The age is the time at which the first scan was taken.

\begin{tabular}{cccc}
\hline Patient ID & Number of Scans & Age & Gender \\
\hline P01 & 2 & 68 & $\mathrm{M}$ \\
P02 & 3 & 71 & $\mathrm{M}$ \\
P03 & 2 & 69 & $\mathrm{M}$ \\
P04 & 3 & 63 & $\mathrm{~F}$ \\
P05 & 5 & 65 & $\mathrm{M}$ \\
P06 & 7 & 68 & $\mathrm{M}$ \\
P06 & 6 & 66 & $\mathrm{M}$ \\
P08 & 5 & 54 & $\mathrm{M}$ \\
P09 & 5 & 62 & $\mathrm{M}$ \\
P10 & 4 & 73 & $\mathrm{M}$ \\
P11 & 59 & $\mathrm{M}$ \\
P12 & 4 & 70 & $\mathrm{M}$ \\
P13 & 6 & 54 & $\mathrm{M}$ \\
P14 & 4 & 72 & $\mathrm{M}$ \\
\hline
\end{tabular}




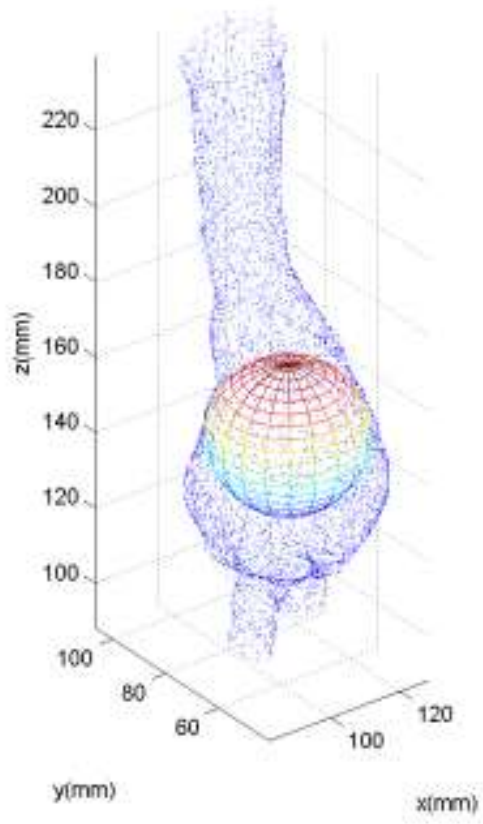

Figure 1. A schematic drawing for illustrating a set of clouded points for the 3D model of an AAA and a maximally inscribed sphere

153 A biomedical software, MIMICS ${ }^{\circledR}$ (Materialize, Leuven, Belgium), is used to reconstruct segmented 3D 154 longitudinal CT data. A smoothing operation is performed after segmentation to sooth down the roughness of the surface resulted from automatic segmentation.

156 Three-dimensional point clouds for the AAA wall with iliac arteries are acquired from the software as an 157 embodiment of the volume of the abdominal aorta (Fig. 1). The point cloud model is essentially a subset 158 of a stereo-lithography (STL) model constructed solely of the vertices of the STL model, which is 159 comprised of four surfaces: two iliac outlet surfaces, one inflow surface at renal level, and the outer wall 160 surface of the abdominal aorta model. 
161 A centerline is a smooth approximation of an infinite series of spherical center points, wherein a 162 maximally inscribed sphere is the largest sphere within the outer arterial wall surface at a centerline point

163 (Fig. 1; [34]). The algorithm of this centerline generation begins by using the centroid of one of the outlet

164 planes as an initial center point guess. Afterwards, the distance from the nearest point in the point cloud 165 and the initial point is computed and the vector connecting these two points is saved. The center of the 166 sphere then moves in the opposite direction of this vector on the outlet plane, within a fixed distance $\delta s$.

167 During the iteration, the position of the centerline is decided when the next position does not change over 16815 iterations. When this process is complete, the first center point $\left(\boldsymbol{p}_{\mathbf{1}}\right)$ is recorded. For the second point $169\left(\boldsymbol{p}_{2}\right)$ we proceed a fixed distance in the direction normal to the outlet plane and repeat the algorithm 170 described previously.

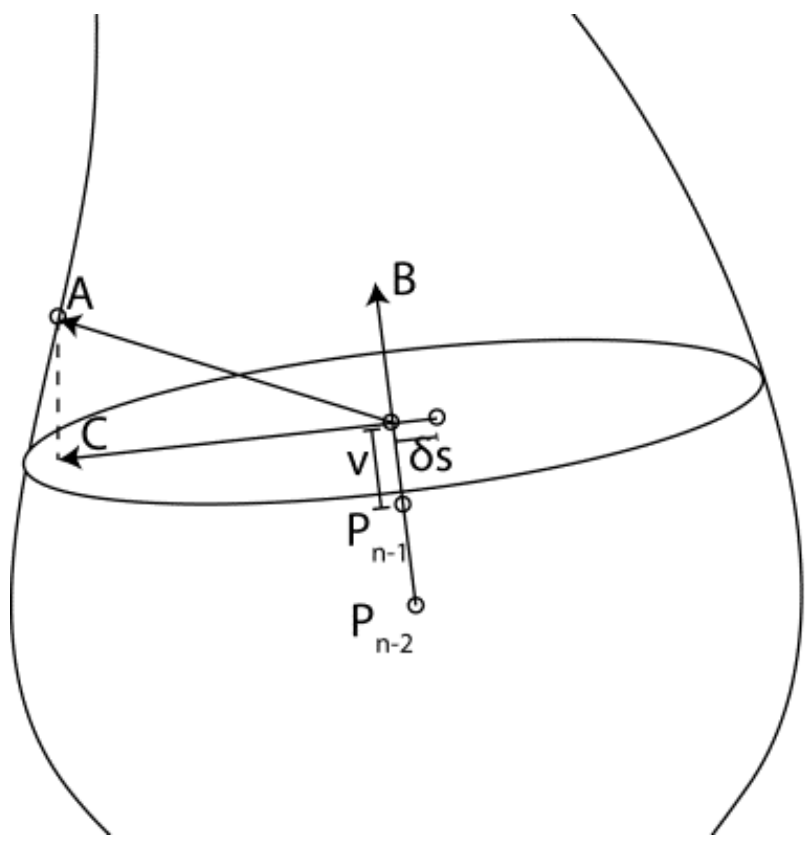

173 Using the previous two successive points, the normal direction $\boldsymbol{B}=\boldsymbol{p}_{\boldsymbol{n}-\mathbf{1}}-\boldsymbol{p}_{\boldsymbol{n}-\mathbf{2}}$ is calculated and serves 174 as an initial translation direction. The new center point translates by $\boldsymbol{B}$ with the magnitude $v$. When the 175 distance from $\boldsymbol{p}_{\boldsymbol{n}}$ to the point on the surface (point cloud) is the minimum value from all points, the vector 
176 from $\boldsymbol{p}_{\boldsymbol{n}}$ to this nearest point is calculated and denoted by $\boldsymbol{A}$, shown in Fig. 2. The vector $\boldsymbol{C}$ is defined by

177 the projection of $\boldsymbol{A}$ onto the plane normal to $\boldsymbol{B}$

$$
C=A-\frac{A \cdot B}{\|B\|^{2}} B
$$

179 The center point $\boldsymbol{p}_{\boldsymbol{n}}$ is then translated in the opposite direction of $\boldsymbol{C}$ by $\delta s$. The iterative process continues 180 and the estimated point keeps translating on the plane (normal to $\boldsymbol{B}$ ). The final $\boldsymbol{p}_{\boldsymbol{n}}$ is determined when the 181 point has not changed by $1 \mathrm{~mm}$ over 15 iterations, and then the algorithm starts again for the next center 182 point. Using the sphere center points and polynomials of fourth order as base functions, a smooth line 183 approximation as a centerline is made. After the centerline is generated, the two bottom cut-planes are 184 determined when the centerlines of two iliac arteries meet at the iliac bifurcation, in which two iliacs are 185 cut normal to their centerlines. Similarly, by using the plane normal to the centerline, the upper cut-plane 186 is determined at the lowest renal level. The algorithm is insensitive to the choice of the chosen outlet cut 187 planes.

188 An orthogonal plane is generated normal to the centerline. An orthogonal diameter $\left(D^{O}\right)$ is the maximum 189 diameter, passing through center point, on each orthogonal plane. Similarly, an axial diameter $\left(D^{A}\right)$ is the 190 maximum diameter on each plane in the axial direction. The sphere diameter is defined as the largest 191 diameter $(D)$ of the maximally inscribed spheres within the AAA volume, and the average of diameter 192 along the aorta $\left(D_{\text {mean }}\right)$ are defined. Using a commercial mesh generation software package for the 193 constructed volume of AAA, the geometry is meshed using tetrahedral elements. The summation of all 194 the volumes of these elements constructs the total volume of the abdominal aortic aneurysm $(V)$ in this 195 work. The extremities of the volume calculation are presented in Fig. 3. 


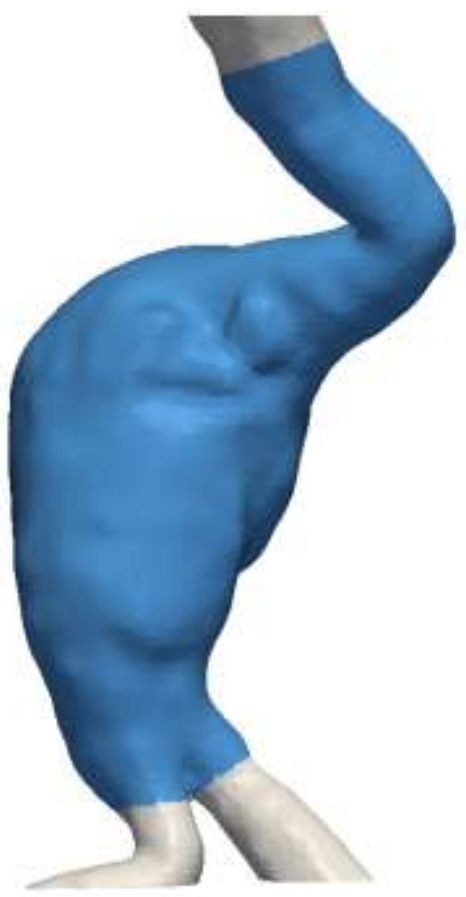
to their respective centerlines.

\section{Growth rate measurements}

201 For the purpose of investigating growth rates in various parameters, the following parameters are 202 employed. Diameter growths are computed at all the center points which are at relatively the same 203 position on the centerline. Fastest and average diameter growth rates over the entire aneurismal sac are 204 denoted by $g_{\max }$ and $g_{\text {mean }}$, respectively. Regarding the appellation for each of diameter measurement 205 methods, growth rates in maximum diameter in inscribed-spherical, orthogonal, and axial diameter 206 are $g_{D}, g_{D(O)}$, and $g_{D(A)}$. The growth average of diameter is called $g_{D(m e a n)}$. Finally, the growth rate in 207 total volume is denoted as $g_{V}$. The following table summarizes all the growth rate parameters presented in 208 with a brief description. For the growth rate calculations the in following sections, only the paired scans 209 with the time interval of more than 6 months are used. 


\begin{tabular}{cc}
\hline Parameter & Description \\
\hline$g_{\max }$ & Fastest growth in the diameter measured by the inscribed-spherical diameter \\
$g_{\text {mean }}$ & Average of the growth rate of the diameter measured by the inscribed-spherical diameter \\
$g_{D}$ & Growth rate of the maximum diameter using the inscribed-spherical diameter \\
$g_{D(\text { mean })}$ & Growth rate of the average of the diameter using inscribed-spherical diameter \\
$g_{V}$ & Growth rate of the aneurysm sac volume \\
$g_{D(O)}$ & Growth rate of the maximum diameter using the orthogonal method \\
$g_{D(A)}$ & Growth rate of the maximum diameter using the axial method \\
$g_{\operatorname{mean}(O)}$ & Average of the growth rate of diameter using the orthogonal method \\
$g_{\operatorname{mean}(A)}$ & Average of growth rate of the diameter measured by the axial method \\
$g_{\max (O)}$ & Fastest growth in diameter measured by the orthogonal method \\
$g_{\max (A)}$ & Fastest growth in diameter measured by the axial method \\
\hline
\end{tabular}

212 A Pearson's correlation $r$ is calculated between all pairs of growth parameters. This analysis was carried 213 out to assess the suitability of different parameters in monitoring the AAA expansion rate. Additionally, 214 median and interquartile ranges (IQR) for growth parameters are calculated. An ANOVA, Shapiro-Wilk 215 tests, and multiple pairwise t-tests are done using $\mathrm{R}$ statistical analysis software (v 3.0.2, R Foundation, 216 Vienna, Austria) with the significance level $\alpha=0.025$. The same significance level is utilized for a 217 correlation study.

\section{Results}

221 Using one scan image, the step sizes $v$ was determined. Individual centerlines were generated with 222 different step sizes and the results found that the maximum $v$ is $6 \mathrm{~mm}$, below which the path of the 223 centerline almost did not change. Considering the computational costs, a $6 \mathrm{~mm}$ of step size is opted for all 224 the study. Regardless, centerline is not sensitive to a higher value of step size. However, for the in-plane 
226 image resolution, the algorithm becomes instable.

\section{Patient P06}

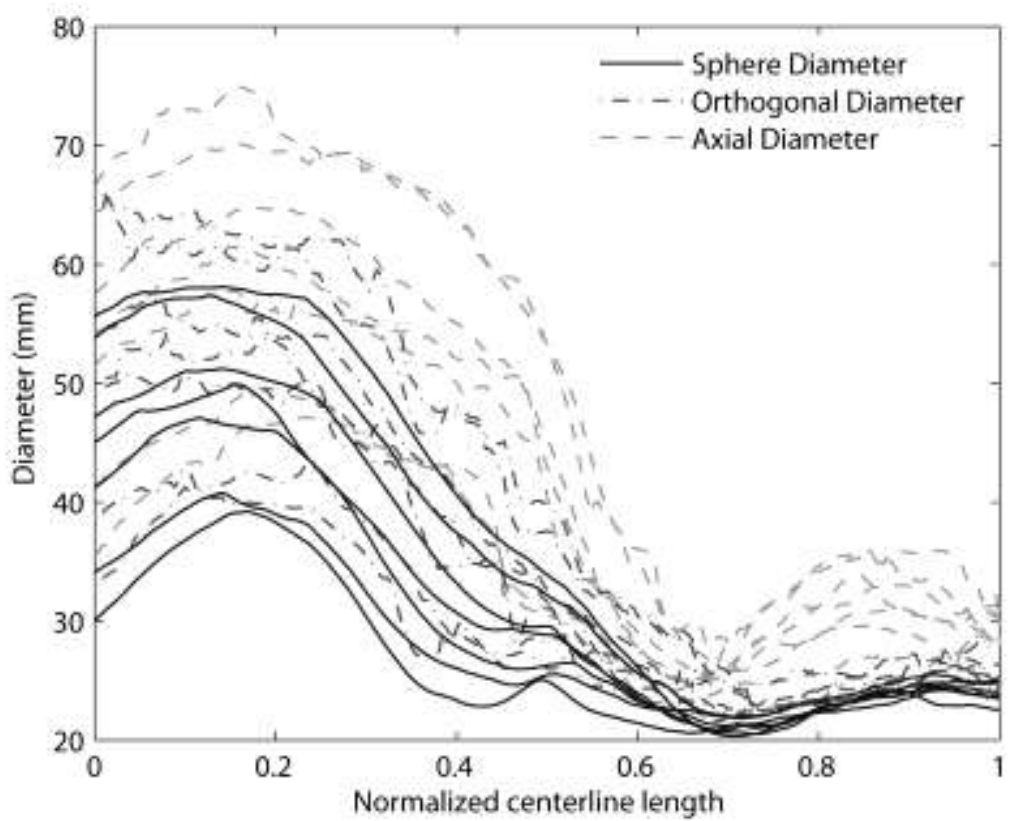

Patient P08

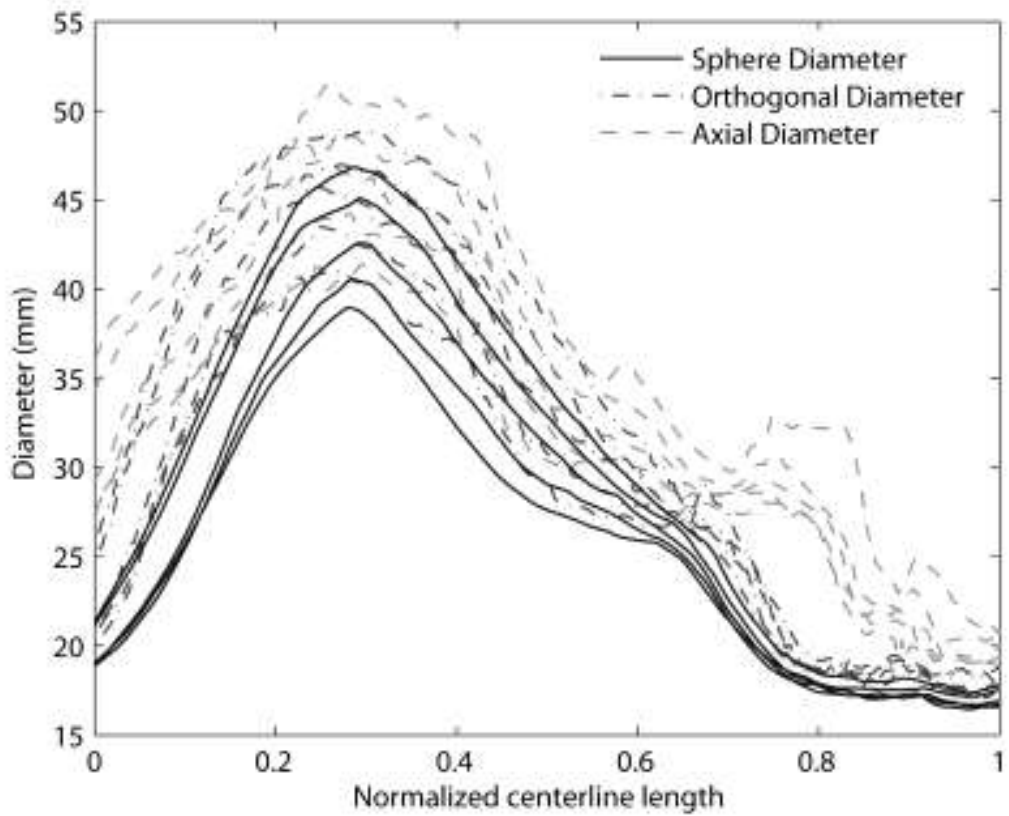




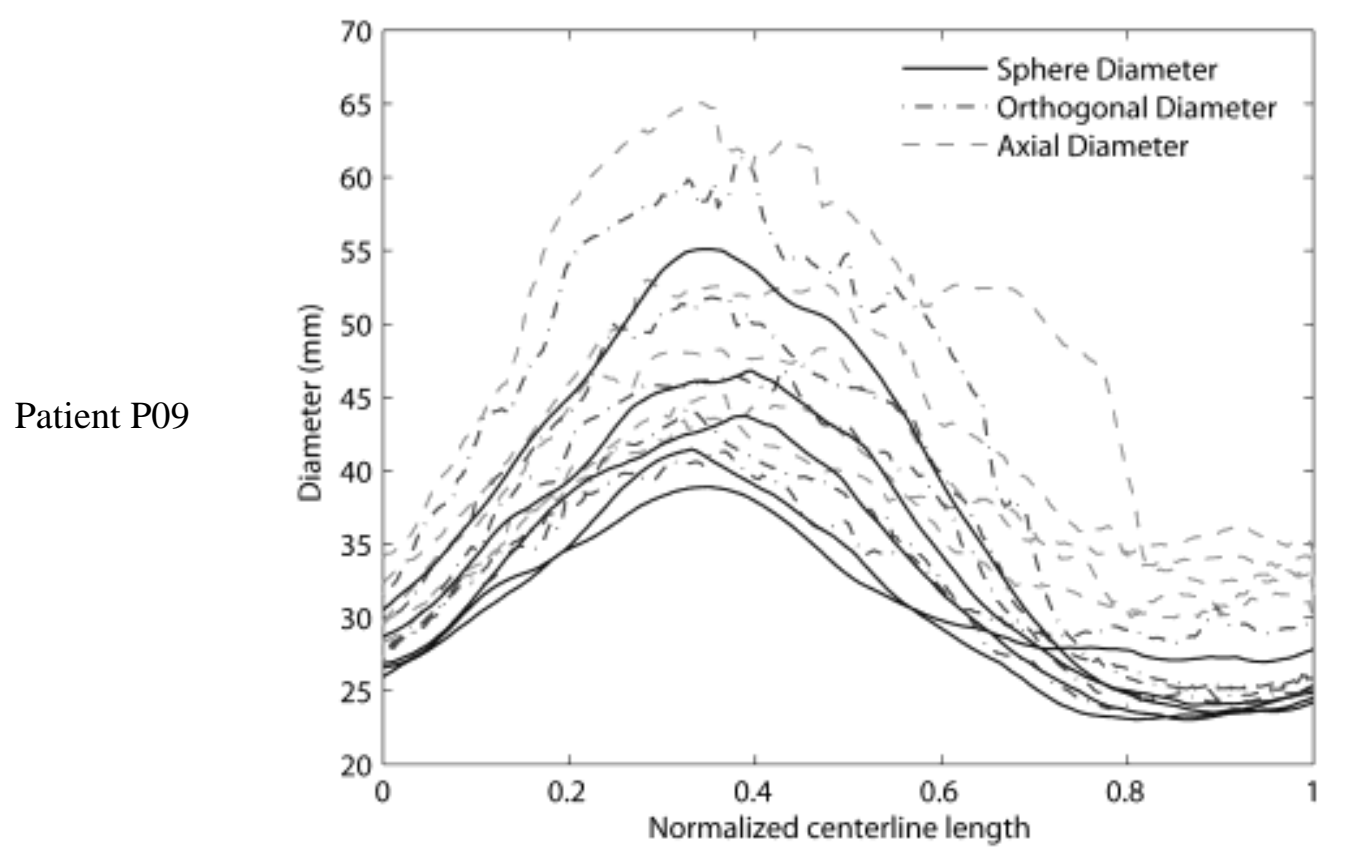

229 Orthogonal, axial, and inscribed-spherical diameters over a normalized centerline for three patients (P06, 230 P08 andP09) are illustrated in Fig. 4. Using the set of the point clouds for patients' images, all of the 231 different diameter measurements are automatically generated. Clearly, the inscribed-spherical diameter 232 shows a smoother curve in comparison with the orthogonal and axial diameters. Also, the plots of the 233 axial diameters show high fluctuations along the normalized centerline length and it appears that the 234 previous plot of the scan can be significantly changed from the next plot. An ANOVA resulted that the 235 difference between maximum diameters measured are different across these three methods. The average 236 and the largest differences between the axial and inscribed-spherical measurements for the maximum 237 diameter among all of the scans are 8 and 18 millimeters $(\mathrm{mm})$ respectively. On the other hand, the 238 difference between the orthogonal and inscribed-spherical measurements for the maximum diameter is on 239 average $4 \mathrm{~mm}$ with a maximum of $16 \mathrm{~mm}$. Finally, the maximum values of the axial and orthogonal 240 diameter measurements vary by $3 \mathrm{~mm}$ on average and by $15 \mathrm{~mm}$ maximally. Both the inscribed-spherical 
241 and orthogonal diameters follow similar patterns in these three patients; but the measured values do not

242 coincide, which naturally contributes to discrepancies in later investigations.

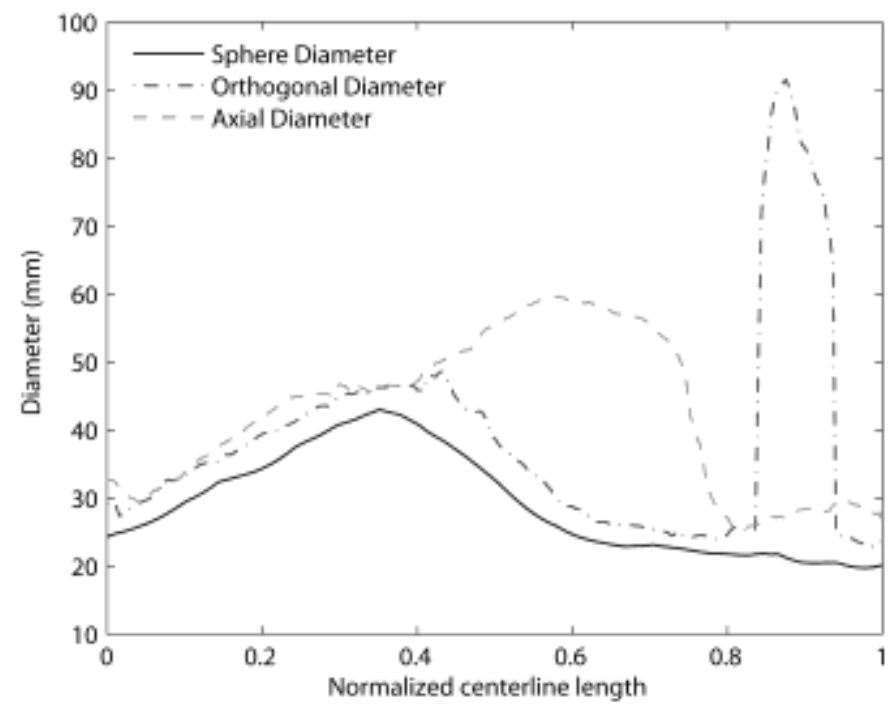

Figure 5. Three diameter measurements for Patient P02-3. The P02-3denotes the third scanned image from patient
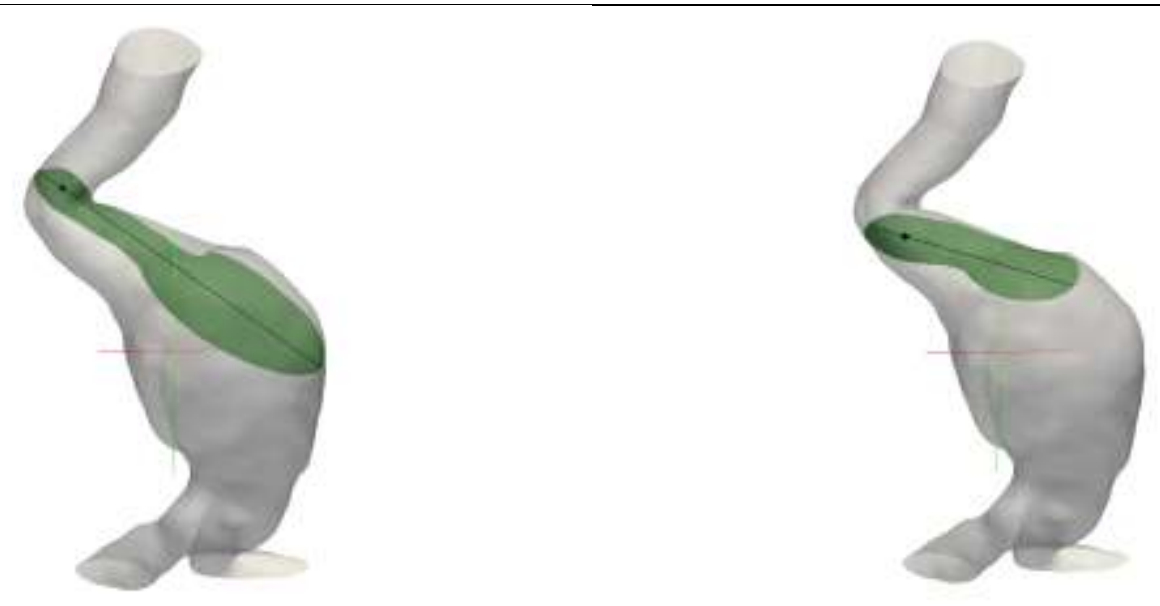

Figure 6. The left panel shows the cross section on orthogonal plane and the right panel shows the cross section on the axial plane. The black dot is the intersection of the centerline with planes. 
The difference between three methods is directly dependent on the shape of the AAA. As an example, for patient $\mathrm{P} 02$, the risk of inaccuracy of automatically measuring the diameter using the orthogonal diameter can be observed (Fig. 5). This happens due to an abrupt change in the direction of the centerline where the aneurysmal neck starts to expand (Fig. 6). Similarly, the axial diameter (the marked region shown the right panel of Fig. 5) is erroneously measuring the AAA diameter over the centerline.
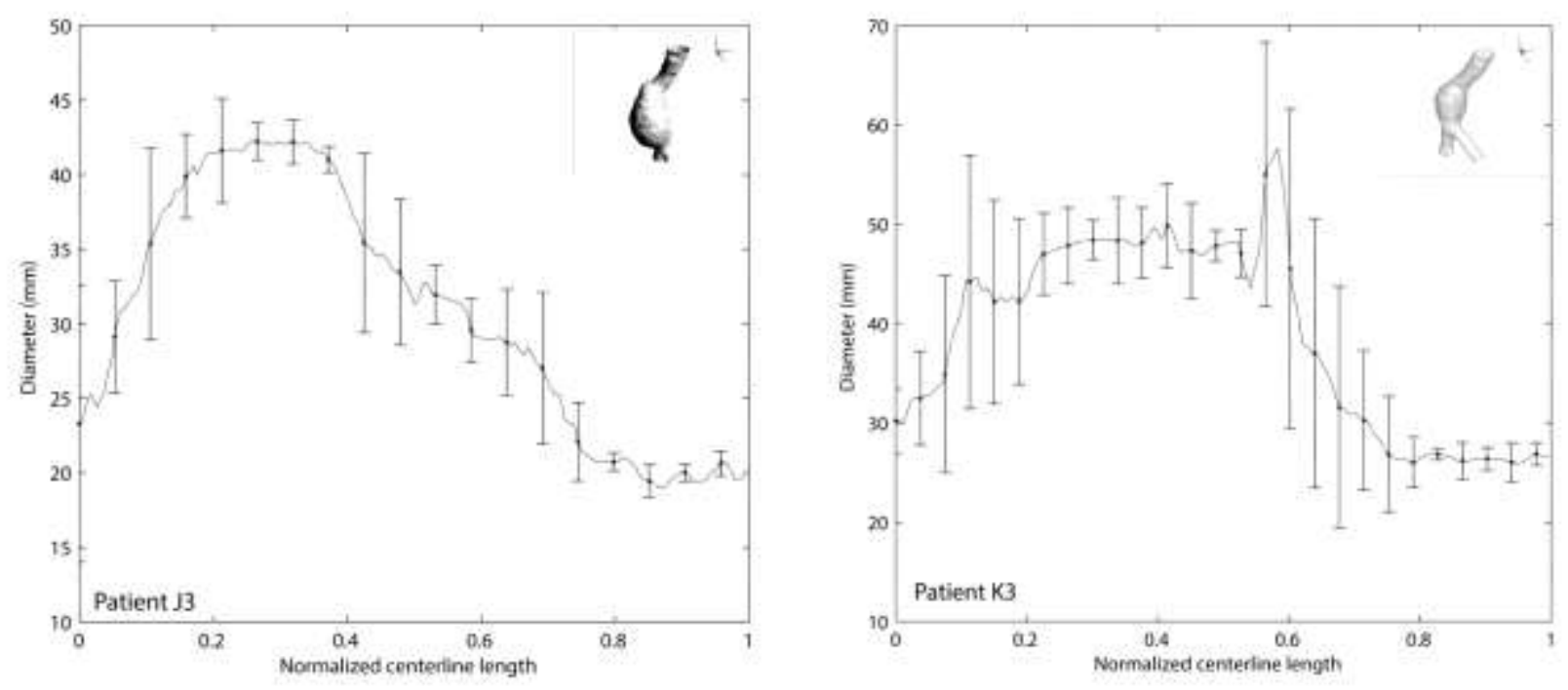

Figure 7. Uncertainty analysis of orthogonal diameter measurement for two different images from the Patients P08and P9

An uncertainty analysis is conducted on the orthogonal diameter method to assess the influence of a small error in finding the orthogonal plane on the calculation of the diameter of an AAA. A set of orthogonal planes is chosen along the centerline and then tilted $5^{\circ}$ in 10 different uniformly discrete directions. The results for the two scans are shown in Figure 7.

The left panel of Figure 7 (Patient P08-3) shows relatively low variability to the orthogonal plane selection, in comparison with the right panel of Figure 7 (Patient P09-3), which displays about a $15 \mathrm{~mm}$ misestimation of a maximum orthogonal diameter. Three dimensional models of AAAs shown on the 

shape of the aneurysm.

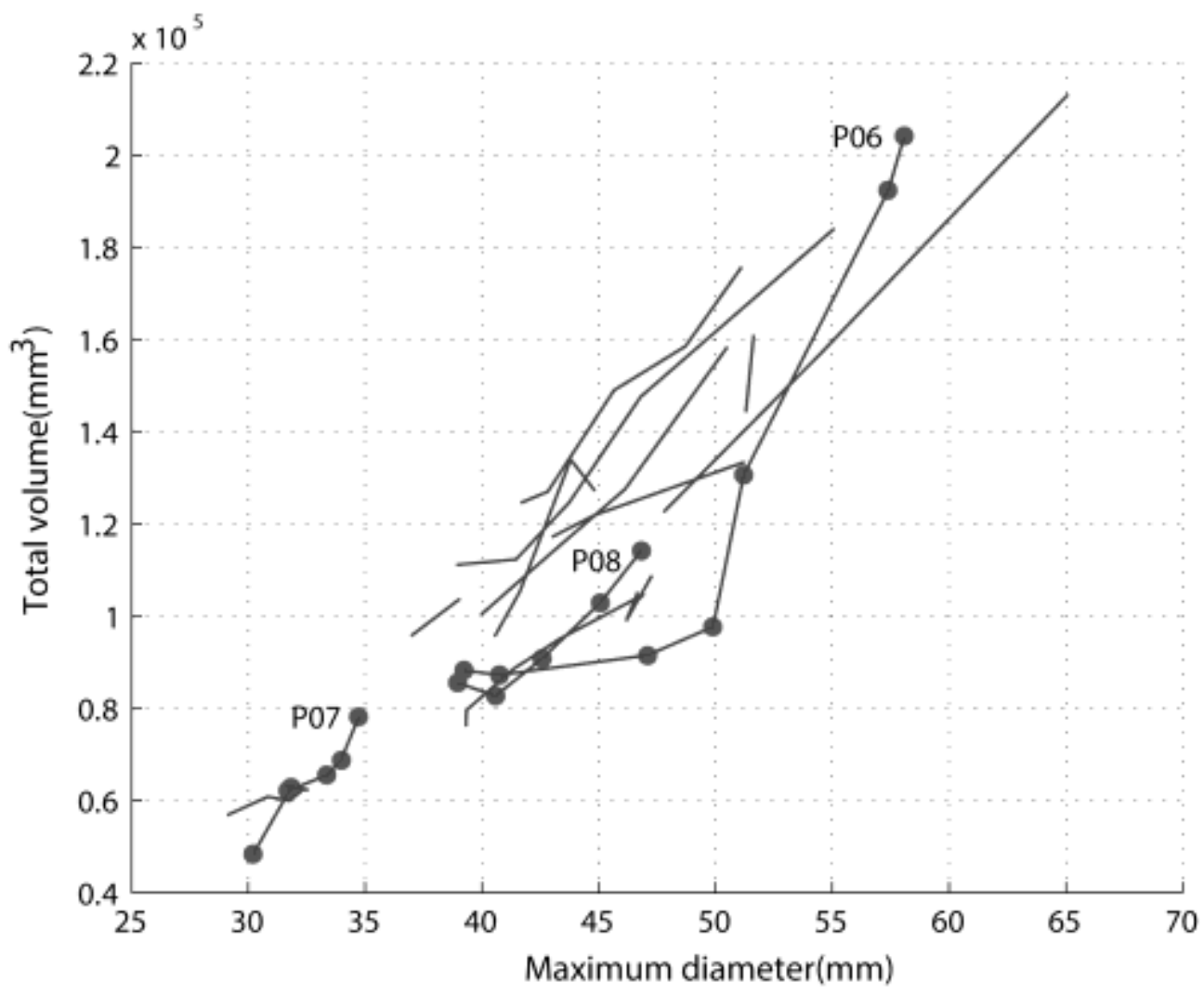

Figure 8. Expectedly there is a positive correlation between maximum inscribed spherical diameter and total volume of

270 The total volumes of AAAs and the maximum inscribed spherical diameters are plotted in Fig. 8 for all

271 the patients studied in this paper. A general trend expresses a continuous growth of the diameter and 272 volume over time, while a small amount of contraction in the volume is seen for some of the patients.

273 Interestingly, the aneurysmal volume of Patient P06 remains constant in the first three images, while the

274 diameter keeps on growing; but a large growth abruptly started after the $4^{\text {th }}$ image was observed. It is 275 worth mentioning that the surveillance period is nearly the same between scan 1-3 and scan 3-6 (almost 276 four years) for this patient. 
Patients P07 and P08, on the other hand, have relatively small changes in volume. Specifically, Patient P07 has one of the smallest AAAs. In addition, the time interval between the third and seventh scan of Patient P06 was approximately 40 months, while Patient P07 was scanned over a period of almost 70 280 months.

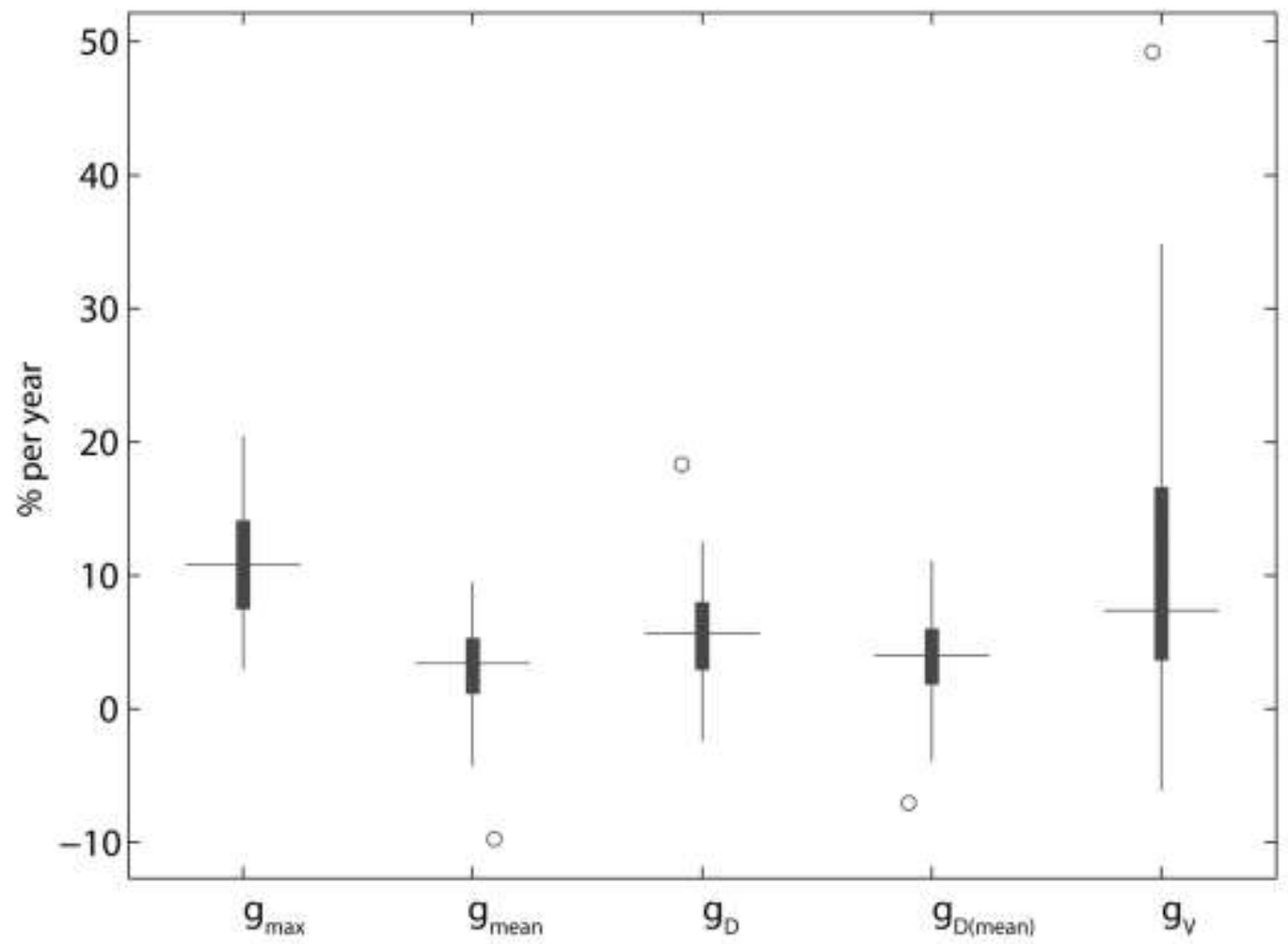

Before presenting the growth rate results, patient P11 was omitted from the statistical analysis due to some irregularities in automatic quantification of orthogonal diameters. A Shapiro-Wilk test on different growth rate parameters confirmed the normality of the data. The computed growth rates in AAA 
IQR $5.00 \%$ /year) is significantly different from the maximum growth rate in diameter $g_{\max }$ (Median 10.69\%/year, IQR $6.61 \% / y e a r)$ with $\mathrm{p}$-value $<0.001$. This denotes that the maximum growth does not necessarily coincide with the point of the normalized centerline length where the aneurysm diameter is

291 the maximum. The average growth rate of diameter is $g_{\text {mean }}$ (Median 3.14\%/year, IQR $\left.4.24 \% / y e a r\right)$, 292 which is also different from $g_{D(\text { mean })}$ (Median $3.74 \% / y e a r$, IQR $4.18 \% /$ year) with p-value $<0.001$. The 293 change in the total volume $g_{V}$ has the largest range amongst all geometrical properties (Median 7.12 $294 \% / y e a r$, IQR 15.60\%/year).

295 The correlation analysis demonstrates that $g_{V}$ is correlated with $g_{D \text { (mean })}(\mathrm{r}=0.48$, p-value $=0.005)$ and $296 g_{\max }(\mathrm{r}=0.53, \mathrm{p}$-value $=0.0015)$, respectively. The correlation between $g_{\text {mean }}$ and $g_{V}(\mathrm{r}=0.40$, $\mathrm{p}-$ 297 value $=0.0205$ ) is not statistically significant, although the small p-value possibly indicates some weak 298 evidence on the correlation. On the other hand, the correlation between $g_{V}$ and $g_{D}(\mathrm{r}=0.53$, $\mathrm{p}$ 299 value $=0.0015)$ is statistically significant based on the p-value. As expected, strong correlations were 300 observed between $g_{\text {mean }}$ and $g_{\max }\left(\mathrm{r}=0.70\right.$, p-value<0.001), $g_{\text {mean }}$ and $g_{D(\text { mean })}(\mathrm{r}=0.99$, $\mathrm{p}-$ 301 value $<0.001)$ and $g_{\text {mean }}$ and $g_{D}(\mathrm{r}=0.60$, p-value<0.001). Furthermore, relatively robust correlations 302 between $g_{\max }$ and $g_{D}(\mathrm{r}=0.75$, p-value $<0.001)$ and $g_{\max }$ and $g_{D(\text { mean })}(\mathrm{r}=0.75$, $\mathrm{p}$-value $<0.001)$ are 303 observed. These correlations and the rest of important correlations between parameters are summarized in 304 the correlation analysis table shown below.

Table 3. Correlations between key growth rate parameters

\begin{tabular}{rcrrrrrrrrr}
\hline & Variable & 1 & 2 & 3 & 4 & 5 & 6 & 7 & 8 & 9 \\
\hline 1 & $g_{\max }$ & 1.00 & & & & & & & & \\
2 & $g_{\text {mean }}$ & 0.70 & 1.00 & & & & & & & \\
3 & $g_{\max (O)}$ & 0.71 & 0.31 & 1.00 & & & & & & \\
4 & $g_{D(\text { mean })}$ & 0.75 & 0.99 & 0.35 & 1.00 & & & & & \\
5 & $g_{D}$ & 0.75 & 0.60 & 0.41 & 0.66 & 1.00 & & & & \\
6 & $g_{D(O)}$ & 0.56 & 0.35 & 0.40 & 0.42 & 0.77 & 1.00 & & & \\
7 & $g_{\max (A)}$ & 0.48 & 0.10 & 0.61 & 0.20 & 0.52 & 0.48 & 1.00 & & \\
8 & $g_{D(A)}$ & 0.53 & 0.39 & 0.22 & 0.47 & 0.72 & 0.76 & 0.49 & 1.00 & \\
9 & $g_{V}$ & 0.53 & 0.40 & 0.28 & 0.48 & 0.53 & 0.54 & 0.53 & 0.60 & 1.00 \\
\hline
\end{tabular}




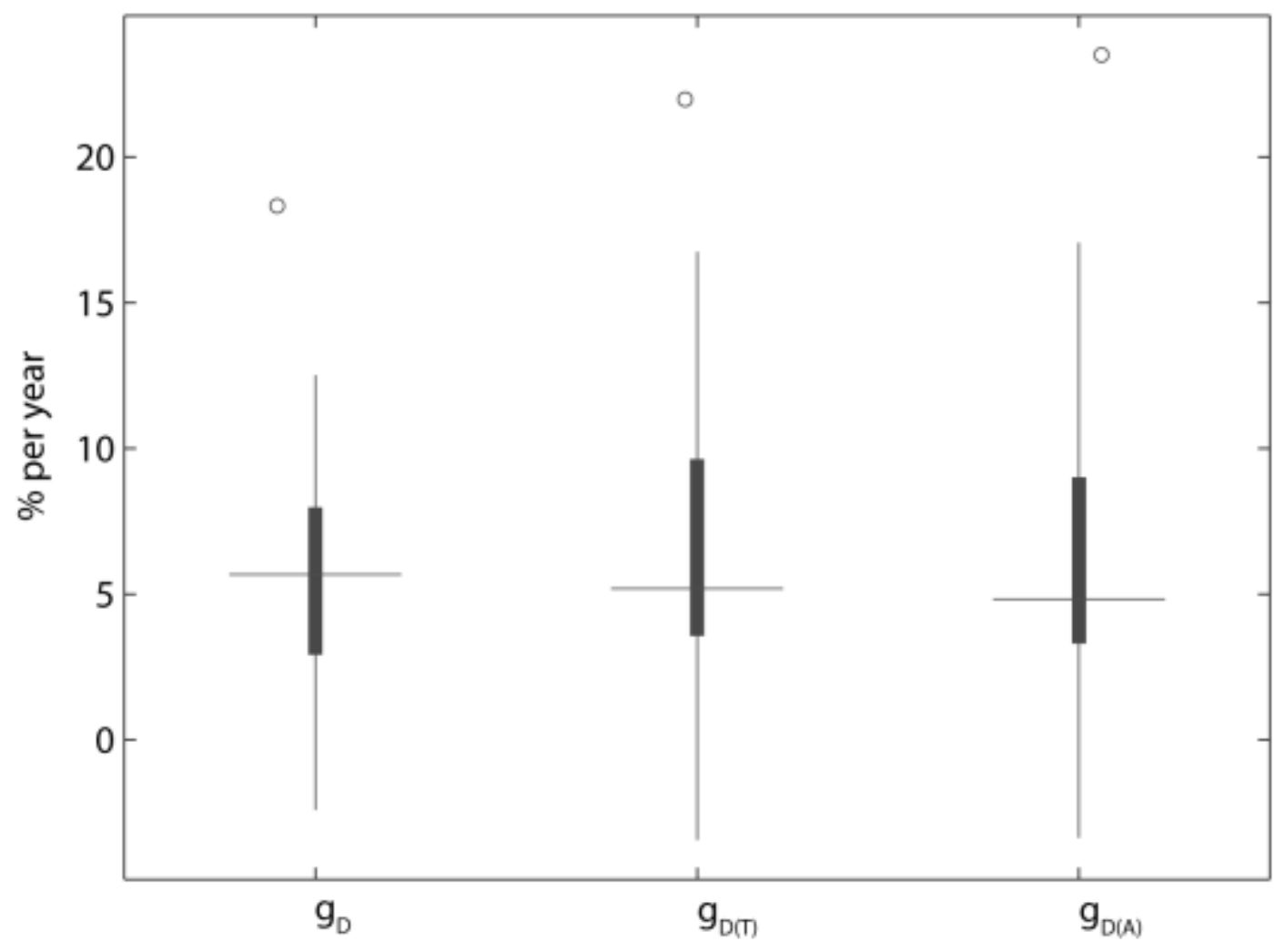

310 Figure 10 compares growth rate measurements for $D, D(O)$ and $D(A)$. Growth rate measurements of the 311 maximum orthogonal and axial diameter, $g_{D(O)}$ (Median $5.08 \% / y e a r$, IQR $\left.6.35 \% / y e a r\right)$ and $g_{D(A)}$ 312 (Median 4.99\%/year, IQR 5.66\%/year) are very similar to that of the inscribed-spherical diameter. There 313 are also correlations between $g_{V}$ and $g_{D(O)}\left(\mathrm{r}=0.54\right.$, p-value=0.001) and between $g_{V}$ and $g_{D(A)}(\mathrm{r}=0.60$, p314 value<0.001). 


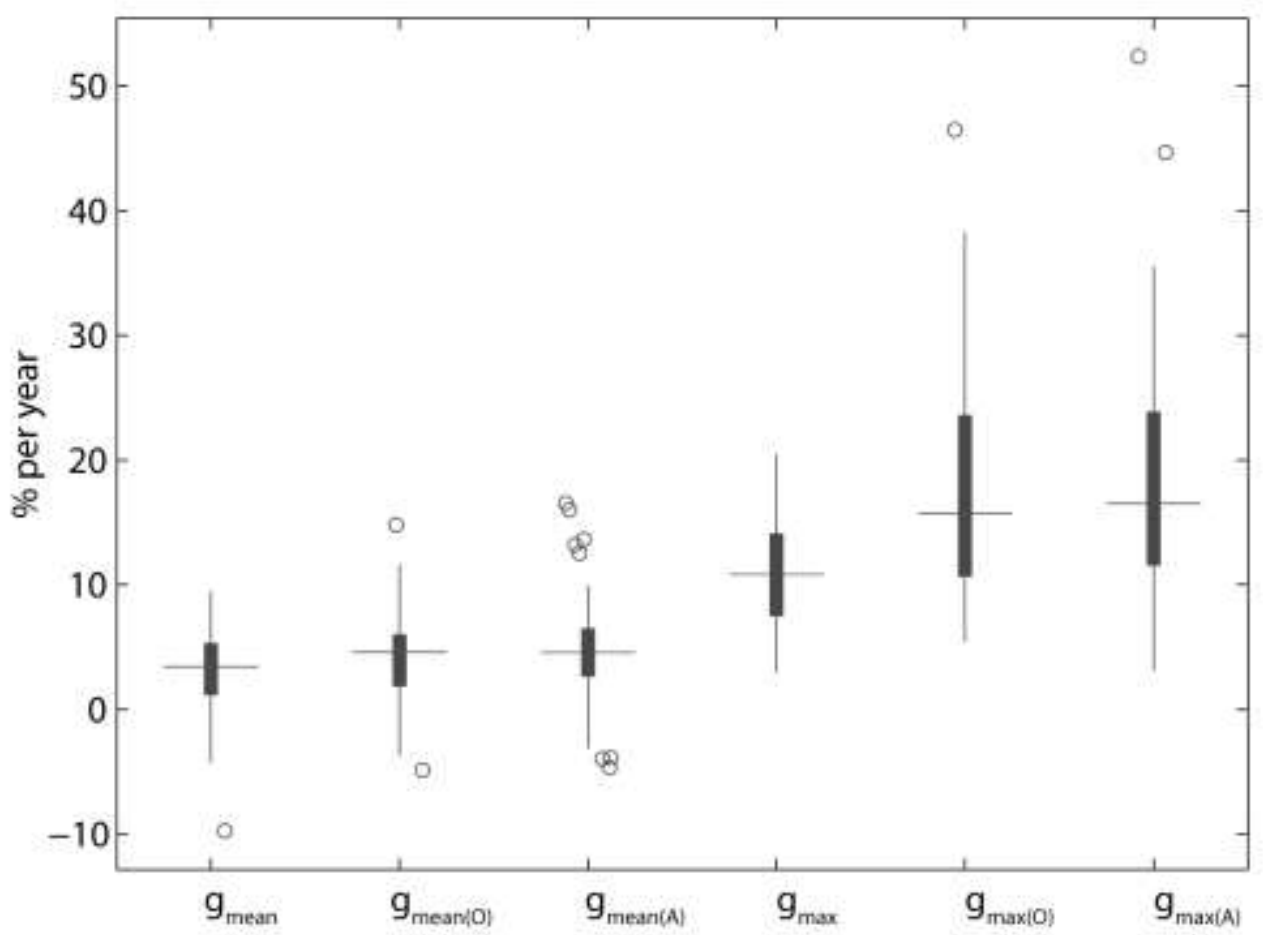

319 Growth rates in the diameter of the aorta for three measurements are compared in Fig. 11. The averages 320 of growth in the diameter are not different for the three measurement methods. Conversely, the fastest 321 growth of the aorta varies considerably for each of the different measurement methods, $g_{\max (O)}($ Median 322 15.69\%/year, IQR $12.79 \% /$ year) and $g_{\max (\mathrm{A})}$ (Median 16.88\%/year, IQR 12.46\%/year). Correlation 323 studies are done between maximal growth of axial, inscribed-spherical, and orthogonal diameter 324 measurements $\left(g_{\max (\mathrm{A})}, g_{\max }, g_{\max (0)}\right)$. It is worth mentioning that no correlation was found between $325 g_{\max (O)}$ and $g_{V}$ in addition to a weak correlation between $g_{\max (O)}$ and $g_{D(O)}(\mathrm{p}$-value $=0.02)$. 


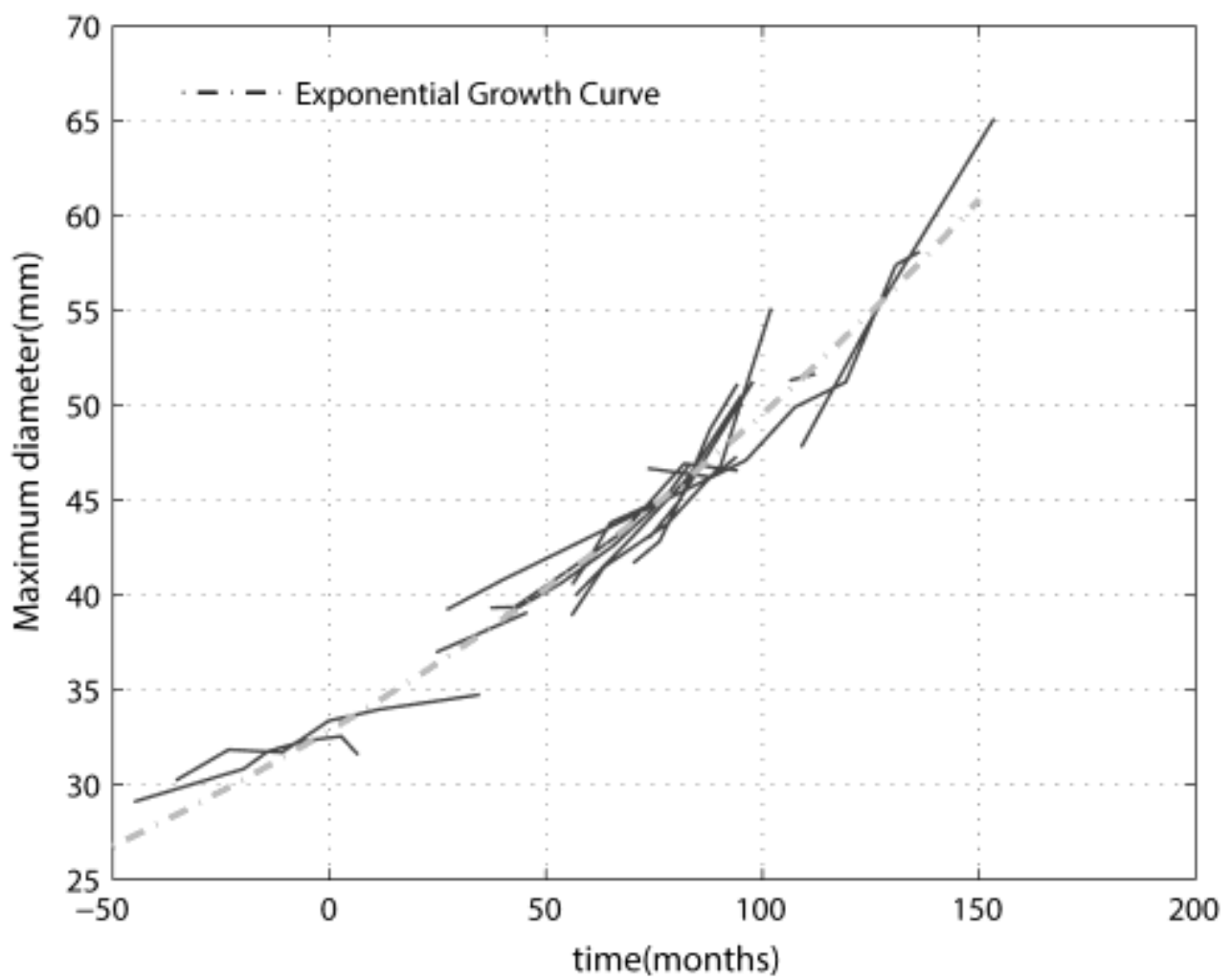

329 Finally, an AAA growth evolution curve is generated with an exponential function for the maximal 330 inscribed-spherical diameter by translating the time axis, shown for all the patients' data in Fig. 12. In the 331 plot, Patient P07, who has been in earlier stages of AAA in comparison with other patients, is in the less 332 steep zone of the curve. In other words, the patient's aneurysm is expanding at a lower rate than a patient 333 in a more advanced stage. The slope of the growth has, in general, a trend of increasing with the time, 334 which is consistent with other studies $[15,16,21,27,29]$. The parameter $g$ found for this curve is $5.06 \% / y$, 335 which is near the median of $g_{D_{\max }}$. 


\section{Discussion}

338 As suggested in multiple studies, orthogonal diameter is a more realistic way to measure the diameter

339 than the diameter measured on the axial plane $[12,13]$. This measurement provides more information

340 about the shape of AAAs, in comparison with other methods, which leads to a more accurate AAA

341 diameter for the therapeutic decisions [35]. Finding the diameter on an orthogonal plane, however, is not

342 a definite procedure [3]. Therefore several replacements have been proposed in different studies

$343[12,13,36]$. For instance, Dillavou et al. [36] suggested that the diameters of the minor axis on the axial

344 planes is more reproducible than the major axis and has the best correlation with orthogonal diameter,

345 thus, it possibly has more applicability for clinical AAA treatment. The inscribed-spherical diameter,

346 introduced in this paper, is a 3D version of the diameter of the minor axis on the orthogonal plane.

347 Apparently, for all AAAs, the mean and maximum diameter is smaller than those of the axial and

348 orthogonal diameter measurements: mean differences between maximum diameters of inscribed-spherical

349 measurement with two others were 8 and $4 \mathrm{~mm}$, respectively.

350 Although, the idea of using a maximally inscribed sphere for generating the centerline is not novel [31-

35133 , it has not been reported in the AAA's diameter measurement before. This method is, hence, applied

352 to AAAs for enhancing its prediction capability, is evaluated with quantification of several parameters,

353 and is studied for the correlation analysis between the parameters. In this paper, this centerline generation

354 algorithm exploits different AAA diameter measurement methods. The results showed their low

355 sensitivity to different variables, such as steps sizes, choice of the outlet planes, and initial guesses.

356 Besides the centerline using this algorithm generation was completely automated.

357 The mean values of growth rates in maximum diameters (e.g. $g_{D}, g_{D(O)}$ and $g_{D(A)}$ ) are not considerably

358 different for the three measurement methods. Nonetheless, the fastest growth of an aorta computed from

359 the inscribed-spherical diameter measurement has the narrowest range among the different measurement

360 methods $\left(g_{\max }, g_{\max (O)}\right.$ and $g_{\max (A)}$ shown in Fig. 11). Both $g_{\max }$ and $g_{\max (A)}$ are highly correlated 
with growth rate of volume. Particularly, these parameters (fastest growth in the diameter and volume) have been associated with structural wall integrity and stress distribution [16,26]. Meanwhile, $g_{\max (O)}$ does not appear to be correlated with $g_{V}$. Besides, only a poor correlation is reported between these two parameters in Martufi et al [16] which reinforces our results. It is worthy to note that the growth rate in the diameter at the exact same position ought to be considered to quantify this parameter which is not practically achievable due to uncertainties in various variables, for example changing posture of the

367 patient during the longitudinal, different scans. In addition, according to what was observed in Figure 4, some of the shape related attributes of the AAA geometry may reflect in quantification of this parameter. These features' effects may vary by the nature of the different measurement methods.

Finding the plane orthogonal to the centerline could also be a source of inaccuracy in orthogonal diameter measurement. Based on the uncertainty analysis, an error of 5 degrees in determining the orthogonal plane can cause up to $15 \mathrm{~mm}$ of miscalculation in measuring the AAA's diameter shown in Fig 7. Finally, in axial and orthogonal diameter measurements, some irregular aneurysmal shapes require an additional manual measurement process, especially if a detailed pointwise quantification of the growth rate of the diameter along the centerline is planned.

Associated with determining surgical interventions and surveillance of interval time, The growth rate

377 calculation using the AAA wall geometry has been proved to be crucial in assessing the risk of rupture $378[2,12,15,16]$. Although a growth pattern captures reasonable AAA growth behavior, shown in Figure 12, 379 growth rates vary for patients, which is believed to be associated with factors such as advanced age, 380 severe cardiac disease, effect of intraluminal thrombus, and a history of tobacco use [37,38]. Nonetheless, 381 monitoring a growth rate of a single criterion like maximum diameter, which is currently used in clinical 382 practice, may not provide sufficient information $[3,16]$.

383 Correlation analysis displays that the growth rate of volume is correlated with the growth rate of 384 maximum diameter using each one of the methods. This relationship shows that monitoring the change of 
maximum diameter over time could lead to finding the growth in volume in cases where the volume's

386 growth is chosen as the major criteria for decision making for a therapeutic procedure. As mentioned

387 before, the growth rate of the volume is correlated with the fastest growth of an aneurysm $\left(g_{\max }\right)$.

388 Additionally, the fastest growth of an aneurysm was found to be fairly correlated with the growth rate of

389 maximum and average diameter. In other words, by finding an expansion rate of the maximum diameter

390 of an AAA, which is more applicable in clinical practice, and an average diameter over the centerline, a

391 prediction of the fastest growth in diameter can be made.

392 The maximally inscribed-sphere method, by its nature, reduces the roughness of the surface of the 393 geometry. As a result of this feature, there are, however, possible shortcomings in terms of the diameter 394 measurement. First, the inscribed-sphere diameter measurement produces lower values as a measure for 395 the size of an AAA. This, in fact, is not very desirable if this diameter measurement is used as a single 396 criterion in clinical practice. Second, this diameter measurement may not be able to capture local 397 expansions on the aneurysm surface. Due to these shortcomings, the maximally inscribed sphere method 398 generates the centerline and the orthogonal diameter measurement is used as a key criterion for the 399 rupture potential, while the inscribed-spherical diameter measurement may be used as a complimentary 400 aid for predicting AAA growth rate. Regardless of these limitations, this method seems to provide 401 valuable information about size related expansion of aneurysms and produces acceptable growth rate 402 parameters with meaningful correlations.

\section{Conclusion}

405 All the measurements used in this study, albeit to different extents, are reliant on the same centerline. 406 Establishment of the centerline, executed completely automatic, showed very low variability to different 407 parameters for instance step size, initial guess and outlet planes. 
408 Using different measurement methods to quantify the diameter of an AAA reflects different values, which

409 may lead to different interpretations from both static and dynamic points of view. While each method has

410 its own unique capabilities and shortcomings, orthogonal measurement seems to remain the gold standard

411 for therapeutic decision making by clinicians. Nevertheless, the inscribed-spherical diameter showed to

412 deliver useful information about the evolution of the size of the abdominal aorta in AAA patients.

413 The exponential growth curve is the empirical model of the evolution of AAA size, here marked by the 414 diameter over time. Using this pattern, the stage of AAA and the future of the AAA expansion can be 415 speculated for each patient. Therefore, future monitoring and treatment procedures can be planned to 416 minimize treatment risks in AAA patients.

\section{Acknowledgement}

420 Authors gratefully acknowledge the support, in part, by the National Heart, Lung, and Blood Instintute of 421 the National Institutes of Health (R01HL115185 and R21HL113857) and National Science Foundation 422 (CMMI-1150376). 


\section{Declaration statements}

426 The contents of solely the responsibility of the authors and does not necessarily represent the official

427 views of the NIH and NSF. We wish to confirm that there are no known conflicts of interest associated

428 with this publication.

429 We declare that we have no significant competing financial, professional or personal interests that might 430 have influenced the performance or presentation of the work described in this manuscript.

431 We confirm that the manuscript has been read and approved by all named authors and that there are no 432 other persons who satisfied the criteria for authorship but are not listed. We further confirm that the order 433 of authors listed in the manuscript has been approved by all of us.

434 We confirm that we have given due consideration to the protection of intellectual property associated with 435 this work and that there are no impediments to publication, including the timing of publication, with 436 respect to intellectual property. In so doing we confirm that we have followed the regulations of our 437 institutions concerning intellectual property.

438 We further confirm that any aspect of the work covered in this manuscript that has involved human 439 patients has been conducted with the ethical approval of Internal Review Board approvals at Michigan 440 State University and Seoul National University Hospital. 


\section{References}

444 1. Doyle BJ, Callanan A, Burke PE, Grace PA, Walsh MT, Vorp DA, et al. Vessel asymmetry as an 445 additional diagnostic tool in the assessment of abdominal aortic aneurysms. J Vasc Surg. 2009; $446 \quad 49: 443-54$.

447 2. Kleinstreuer C, Li Z. Analysis and computer program for rupture-risk prediction of abdominal aortic 448

3. Vande Geest JP, Wang DHJ, Wisniewski SR, Makaroun MS, Vorp DA. Towards a noninvasive method for determination of patient-specific wall strength distribution in abdominal aortic aneurysms. Ann Biomed Eng. 2006; 34:1098-106.

4. Lederle FA, Wilson SE, Johnson GR, Reinke DB, Littooy FN, Acher CW, et al. Variability in measurement of abdominal aortic aneurysms. Abdominal Aortic Aneurysm Detection and Management Veterans Administration Cooperative Study Group. J Vasc Surg. 1995; 21:945-52.

5. Lederle FA, Wilson SE, Johnson GR, Reinke DB, Littooy FN, Acher CW, et al. Immediate repair compared with surveillance of small abdominal aortic aneurysms. N Engl J Med. 2002;346:143744.

6. Lederle FA, Johnson GR, Wilson SE, et al. Rupture rate of large abdominal aortic aneurysms in patients refusing or unfit for elective repair. J Am Med Assoc. 2002; 287:2968-72.

7. Brown PM, Zelt DT, Sobolev B. The risk of rupture in untreated aneurysms: The impact of size, gender, and expansion rate. J Vasc Surg. 2003; 37:280-4.

8. Nevitt MP, Ballard DJ, Hallett JW. Prognosis of Abdominal Aortic Aneurysms. N Engl J Med. 1989; 321:1009-14.

9. The UK Small Aneurysm Trial Participants. Long-Term Outcomes of Immediate Repair Compared with Surveillance of Small Abdominal Aortic Aneurysms. N Engl J Med. 2002; 346:1445-52.

10. The UK Small Aneurysm Trial Participants. Mortality results for randomised controlled trial of early elective surgery or ultrasonographic surveillance for small abdominal aortic aneurysms. The UK Small Aneurysm Trial Participants. Lancet. 1998; 352:1649-55.

11. Abada HT, Sapoval MR, Paul JF, de Maertelaer V, Mousseaux E, Gaux JC. Aneurysmal sizing after endovascular repair in patients with abdominal aortic aneurysm: interobserver variability of various measurement protocols and its clinical relevance. Eur Radiol. 2003; 13:2699-704.

12. Kontopodis N, Metaxa E, Gionis M, Papaharilaou Y, Ioannou CV. Discrepancies in determination of abdominal aortic aneurysms maximum diameter and growth rate, using axial and orthogonal computed tomography measurements. Eur J Radiol. 2013; 82; 1398-403.

13. Dugas A, Therasse E, Kauffmann C, Tang A, Elkouri S, Nozza A, Giroux MF, Oliva VL, Soulez G. Reproducibility of abdominal aortic aneurysm diameter measurement and growth evaluation on axial and multiplanar computed tomography reformations. Cardiovasc Intervent Radiol. 2012; 35: 779-87. 
14. Long A, Rouet L, Lindholt JS, Allaire E. Measuring the maximum diameter of native abdominal aortic aneurysms: review and critical analysis. Eur J Vasc Endovasc Surg. 2012; 43: 515-24.

15. Limet R, Sakalihassan N, Albert A. Determination of the expansion rate and incidence of rupture of abdominal aortic aneurysms. J Vasc Surg. 1991; 14: 540-8.

16. Martufi G, Auer M, Roy J, Swedenborg J, Sakalihasan N, Panuccio G, et al. Multidimensional growth measurements of abdominal aortic aneurysms. J Vasc Surg. 2013; 58: 748-755.

17. Darling RC. Ruptured arteriosclerotic abdominal aortic aneurysms: A pathologic and clinical study. Am J Surg. 1970; 119: 397-401.

18. Wever JJ, Blankensteijn JD, Th M Mali WP, Eikelboom BC. Maximal aneurysm diameter followup is inadequate after endovascular abdominal aortic aneurysm repair. Eur J Vasc Endovasc Surg. 2000; 20: 177-82.

19. Nicholls SC, Gardner JB, Meissner MH, Johansen KH. Rupture in small abdominal aortic aneurysms. J Vasc Surg. 1998; 28: 884-8.

20. Schewe CK, Schweikart HP, Hammel G, Spengel FA, Zöllner N, Zoller WG. Influence of selective management on the prognosis and the risk of rupture of abdominal aortic aneurysms. Clin Investig. 1994; 72: 585-91.

21. Cronenwett JL, Murphy TF, Zelenock GB, Whitehouse WM Jr, Lindenauer SM, Graham LM, Quint LE, Silver TM, Stanley JC. Actuarial analysis of variables associated with rupture of small abdominal aortic aneurysms. Surgery. 1998; 98: 472-83.

22. Forbes TL, Lawlor DK, DeRose G, Harris KA. Gender Differences in Relative Dilatation of Abdominal Aortic Aneurysms. Ann Vasc Surg. 2006; 20: 564-8.

23. Hatakeyama T, Shigematsu H, Muto T. Risk factors for rupture of abdominal aortic aneurysm based on three-dimensional study. J Vasc Surg. 2001; 33: 453-61.

24. Baumueller S, Nguyen TDL, Goetti RP, Lachat M, Seifert B, Pfammatter T, Frauenfelder T. Maximum Diameter Measurements of Aortic Aneurysms on Axial CT Images After Endovascular Aneurysm Repair: Sufficient for Follow-up? Cardiovasc Intervent Radiol. 2011; 34: 1182-9.

25. Van Prehn J, van der Wal MBA, Vincken K, Bartels LW, Moll FL, van Herwaarden JA. Intra- and Interobserver Variability of Aortic Aneurysm Volume Measurement With Fast CTA Postprocessing Software. J Endovasc Ther. 2008; 15: 504-10.

26. Raghavan ML, Vorp DA, Federle MP, Makaroun MS, Webster MW. Wall stress distribution on three-dimensionally reconstructed models of human abdominal aortic aneurysm. J Vasc Surg. 2000; 31: 760-9.

27. Vega de Céniga M, Gómez R, Estallo L, Rodríguez L, Baquer M, Barba A. Growth rate and associated factors in small abdominal aortic aneurysms. Eur J Vasc Endovasc Surg Off J Eur Soc Vasc Surg. 2006; 31: 231-6.

28. Hirose Y, Hamada S, Takamiya M. Predicting the growth of aortic aneurysms: a comparison of linear vs exponential models. Angiology. 1995; 46: 413-9. 
29. Grimshaw GM, Thompson JM. The abnormal aorta: a statistical definition and strategy for monitoring change. Eur J Vasc Endovasc Surg Off J Eur Soc Vasc Surg. 1995; 10: 95-100.

30. Vardulaki KA, Prevost TC, Walker NM, Day NE, Wilmink ABM, Quick CRG, et al. Growth rates and risk of rupture of abdominal aortic aneurysms. Br J Surg. 1998; 85: 1674-80.

31. Antiga L, Steinman DA. Robust and objective decomposition and mapping of bifurcating vessels. IEEE Trans Med Imaging. 2004; 23:704-13.

32. Dey TK, Zhao W. Approximate medial axis as a Voronoi subcomplex. Comput-Aided Des. 2004; 36:195-202.

33. Antiga L, Piccinelli M, Botti L, Ene-Iordache B, Remuzzi A, Steinman DA. An image-based modeling framework for patient-specific computational hemodynamics. Med Biol Eng Comput. 2008; 46:1097-112.

34. Dupay AC. Investigation of surface evolution for abdominal aortic aneurysms interacting with surrounding tissues. (Master's thesis). Michigan State University; 2012.

35. Ijaz A. Truncated Gaussian process regression for predicting growth of abdominal aortic aneurysm and for temporal modeling of sentiments. (Master's thesis). Michigan State University; 2013.

36. Dillavou ED, Buck DG, Muluk SC, Makaroun MS. Two-Dimensional Versus Three-Dimensional CT Scan for Aortic Measurement. J Endovasc Ther. 2003; 10: 531-8.

37. Chang JB, Stein TA, Liu JP, Dunn ME. Risk factors associated with rapid growth of small abdominal aortic aneurysms. Surgery. 1997; 121: 117-22.

38. Helderman F, Manoch IJ, Breeuwer M, Kose U, Boersma H, van Sambeek MRHM, et al. Predicting patient-specific expansion of abdominal aortic aneurysms. Eur J Vasc Endovasc Surg Off J Eur Soc Vasc Surg. 2010; 40: 47-53.

39. Poon JTC, Cheng SWK, Wong JSW, Ting ACW. Prevalence of abdominal aortic aneurysm in Chinese patients with severe coronary artery disease. ANZ J Surg. 2010; 80: 630-3.

40. Poon JTC, Cheng SWK, Wong JSW, Ting ACW. Prevalence of abdominal aortic aneurysm in Chinese patients with severe coronary artery disease. ANZ J Surg. 2010 Sep;80(9):630-3. 